\title{
Effect of Crystal Symmetry on the Spin States of Fe3+ and Vibration Modes in Lead-free Double- Perovskite Cs2AgBi(Fe)Br-6
}

Yuttapoom Puttisong, Fabrizio Moro, Shula Chen, Pontus Höjer, Weihua Ning, Feng Gao, Irina Buyanova and Weimin Chen

The self-archived postprint version of this journal article is available at Linköping University Institutional Repository (DiVA):

http://urn.kb.se/resolve?urn=urn:nbn:se:liu:diva-168273

N.B.: When citing this work, cite the original publication.

Puttisong, Y., Moro, F., Chen, S., Höjer, P., Ning, W., Gao, F., Buyanova, I., Chen, W., (2020), Effect of Crystal Symmetry on the Spin States of Fe3+ and Vibration Modes in Lead-free Double-Perovskite Cs2AgBi(Fe)Br-6, Journal of Physical Chemistry Letters, 11(12), 4873-4878.

https://doi.org/10.1021/acs.jpclett.oc01543

Original publication available at:

https://doi.org/10.1021/acs.jpclett.oc01543

Copyright: American Chemical Society

http://pubs.acs.org/ 


\title{
Effect of Crystal Symmetry on the Spin States of $\mathrm{Fe}^{3+}$ and Vibration Modes in Lead-free Double Perovskite $\mathrm{Cs}_{2} \mathrm{AgBi}(\mathrm{Fe}) \mathrm{Br}_{6}$
}

Y. Puttisong ${ }^{\S *}$, F. Moro ${ }^{\S, \uparrow}$, S.L. Chen, P. Höjer, W. Ning, F. Gao, I.A. Buyanova, and W.M. Chen

Department of Physics, Chemistry, and Biology (IFM), Linköping University, SE-58183 Linköping, Sweden

$\S$ These authors contribute equally

${ }^{\dagger}$ Present address: Department of Materials Science, University of Milano-Bicocca, via R. Cozzi 55, 20125, Milan, Italy

*e-mail address: yuttapoom.puttisong@liu.se

\begin{abstract}
We show by electron spin resonance (ESR) and Raman spectroscopies that the crystal phase transition of the lead-free double perovskite $\mathrm{Cs}_{2} \mathrm{AgBiBr}_{6}$ has a profound symmetry-breaking effect on the high-spin states of e.g. a transitional metal ion $\mathrm{Fe}^{3+}$ and the vibrational modes. It lifts their degeneracy when the crystal undergoes the cubic-tetragonal phase transition, splitting the 6 -fold degenerate $\mathrm{S}=5 / 2$ state of $\mathrm{Fe}^{3+}$ to three Kramer doublets, and the enharmonic breathing mode $T_{g}$ of the $\mathrm{MBr}_{6}$ octahedra $(\mathrm{M}=\mathrm{Ag}, \mathrm{Bi}, \mathrm{Fe})$ into $E_{g}+A_{g}$. The magnitudes of both spin and Raman line splitting are shown to directly correlate with the strength of the tetragonal strain field. This work in turn demonstrates the power of the ESR and Raman spectroscopies in probing structural phase transitions and in providing in-depth information on the interplay between the structural, spin and vibrational properties of lead-free double perovskites - a newly-emerging and promising class of materials for low-cost and high-efficiency photovoltaics and optoelectronics.
\end{abstract}


In recent years solution-processed perovskite semiconductors have attracted great attention as being emerging low-cost competitors of Si-based solar cells ${ }^{1-3}$. Indeed, perovskites represent the fastest growing materials system in the context of photovoltaics and light-emitting device applications ${ }^{4-6}$. Power conversion efficiency (PCE) of lead halide perovskite monolithic tandem solar cells has reached $25.9 \%$ in just 10 years $^{3,7}$, thus fairly competing with the traditional solar cells based on crystalline silicon, cadmium telluride, gallium arsenide and copper indium gallium selenide ${ }^{2,3}$. A monolithic tandem Si/perovskite solar cells has reached PCE of $25.1 \%$, demonstrating high compatibility to integrate perovskites with the existing mature Si technology ${ }^{8}$.

Major concerns facing large-scale commercial applications of hybrid lead halide perovskite devices are their toxic nature and instabilities ${ }^{9,10}$, which have motivated intense research efforts in searching for a new class of lead-free perovskites with better stability. By replacing two $\mathrm{Pb}^{2+}$ by a pair of non-toxic $\mathrm{M}^{+}$and $\mathrm{M}^{3+}$ metal ions, the resulting double perovskites with a general formula $\mathrm{A}_{2} \mathrm{M}^{+} \mathrm{M}^{3+} \mathrm{X}_{6}$ ( $\mathrm{A}$ and $\mathrm{M}$ are cations and $\mathrm{X}$ is a halide anion, also known as elpasolite) have emerged as an environmentally friendly alternative to lead perovskites for solar cells ${ }^{11-17}$ as well as for their potential applications as X-ray detectors ${ }^{18}$ and light-emitting diodes ${ }^{19}$. Among them, inorganic $\mathrm{Cs}_{2} \mathrm{AgBiBr}_{6}$ has attracted particular attention because it is highly stable and it shows interesting physical properties like tunable bandgap $^{13,20}$, long carrier recombination lifetimes ${ }^{12}$, long electron-hole diffusion length ${ }^{21}$, excitonic effects and electron-phonon coupling ${ }^{22,23}$. Its functionalities for optoelectronic devices have just started to be explored. Unfortunately, the solar cell performance of $\mathrm{Cs}_{2} \mathrm{AgBiBr}_{6}$, with $\mathrm{PCE}$ reaching $2.51 \%$, is far from competing with the lead-based perovskites ${ }^{16}$. This calls for a better understanding of the fundamental properties of $\mathrm{Cs}_{2} \mathrm{AgBiBr}_{6}$ and related double perovskites including structural, electronic, optical and phonon properties, as well as interactions between them. This is especially important for 

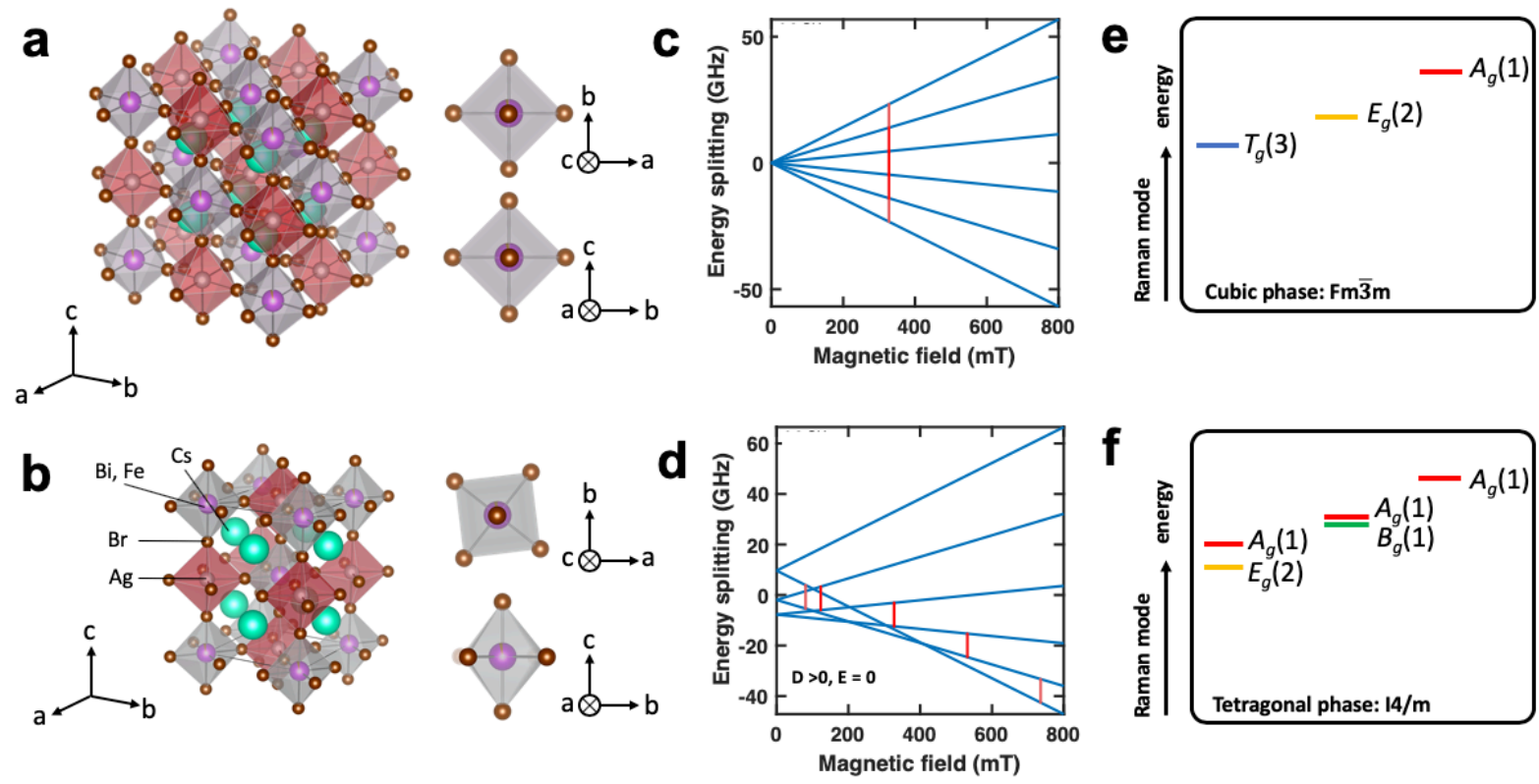

Figure 1 a) and b) crystal structure of $\mathrm{Cs}_{2} \mathrm{AgBiBr}_{6}$ in the cubic and tetragonal phases. The insets show the orientation of the $\mathrm{BiBr}_{6}$ (or $\mathrm{FeBr}_{6}$ in Fe-doped crystal) octahedral sublattices along the $\mathrm{c}$ and a crystallographic axes. c) and d) The predicted spin splitting patterns of $\mathrm{Fe}^{3+}$ (the blue lines) in a magnetic field and the expected ESR transitions at $9.3 \mathrm{GHz}$ (the red vertical lines) in the cubic and tetragonal phases. e) and f) The Raman modes of the MBr6 octahedra predicted for the cubic and tetragonal crystal symmetry.

double perovskites as their physical properties are expected to be considerably different from their lead-based counterparts and are more complex due to the interplay between two different sublattices. The gained knowledge on these fundamental properties is essential for designing strategies to tune optoelectronic properties and to identify new compounds of the same class and their low-dimensional counterparts with tailored physical properties for optimal device performance. Indeed, a structural phase transition of $\mathrm{Cs}_{2} \mathrm{AgBiBr}_{6}$, from a cubic phase (space group Fm $\overline{3} \mathrm{~m}$ - figure 1a) at room temperature to a tetragonal phase (space group I4/m - figure 1b) at temperatures lower than the phase transition temperature $T_{s} \sim 122$ $\mathrm{K}$, has recently been uncovered from studies of X-ray and neutron diffraction ${ }^{24}$. The local symmetry around $\left(\mathrm{BiBr}_{6}\right)^{-3}$ octahedra reduces from cubic (with the same $\mathrm{Bi}-\mathrm{Br}$ bond length along the $\mathrm{a}, \mathrm{b}$ and $\mathrm{c}$ crystallographic axes) to axial (with a larger $\mathrm{Bi}-\mathrm{Br}$ bond length along the $\mathrm{c}$ axis than those in the ab plane in addition to a slight twist of the octahedra around the c axis). This structural change was shown to lead to noticeable changes in optical properties monitored in reflectivity, photoluminescence and absorbance measurements, e.g., the 
excitonic transition energy near the direct gap shifts proportionally to the strength of tetragonal $\operatorname{strain}^{24}$.

In this work, we examine the effects of crystal phase transition on spin states of transition metal ions such as $\mathrm{Fe}^{3+}$ and vibration modes of $\mathrm{Cs}_{2} \mathrm{AgBiBr}_{6}$ by employing electron spin resonance (ESR) and Raman spectroscopy. We show that the transition from the cubic to tetragonal crystal phase lifts the degeneracy of the $\mathrm{S}=5 / 2$ spin states of $\mathrm{Fe}^{3+}$ into three Kramer doublets $\left(\mathrm{M}_{\mathrm{s}}= \pm \frac{1}{2}, \pm \frac{3}{2}\right.$ and $\left.\pm \frac{5}{2}\right)$ at zero magnetic field with the corresponding zerofield spin splitting scaling with the strength of the tetragonal strain. We also reveal that the crystal symmetry-lowering results in splitting of the $T_{2 g}$ vibration mode, and again the extent of the splitting critically depends on the strain field. Our results in turn demonstrate the power of ESR and Raman spectroscopies to sensitively probe the crystal structural change and the strength of the crystal field.
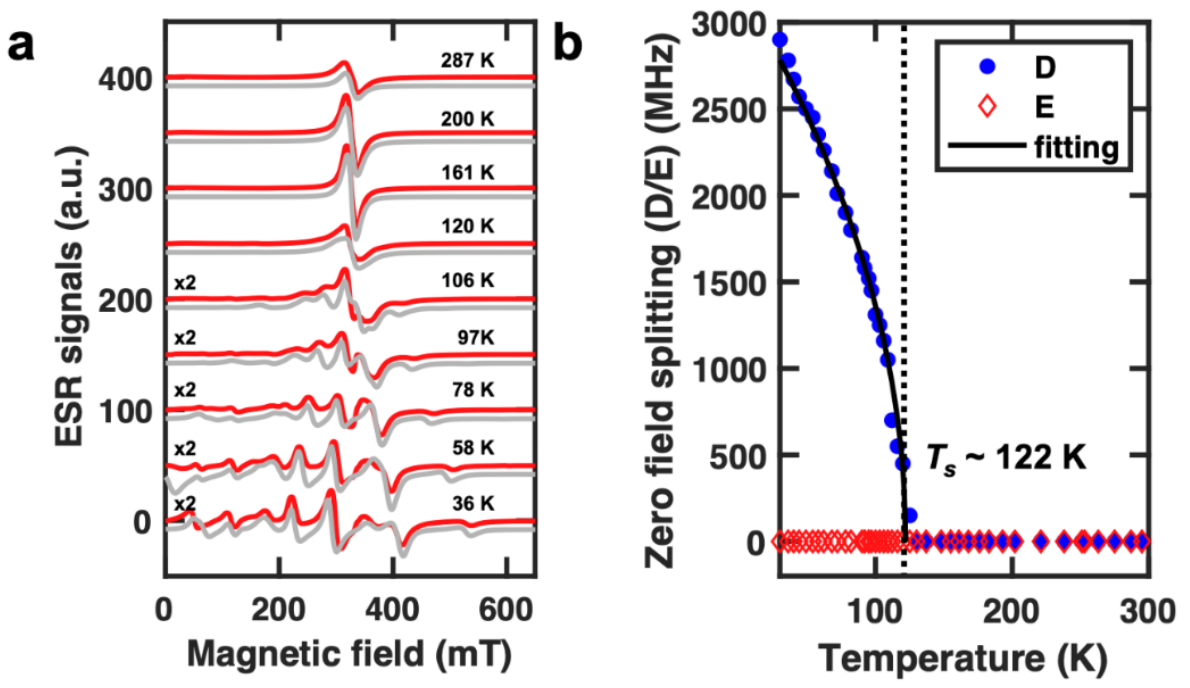

Figure 2 a) Temperature dependent ESR spectra of the Fe-doped $\mathrm{Cs}_{2} \mathrm{AgBiBr}_{6}$ crystal power associated with $\mathrm{Fe}^{3+}$. The red lines are experimental data and the grey lines are the simulated curves from the spin Hamiltonian analysis. b) Temperature dependence of the axial $(D)$ and rhombic $(E)$ zero-field splitting parameters deduced from the ESR spectra of $\mathrm{Fe}^{3+}$. The solid line is the fitting curve using the critical exponents function Eq.2.

Typical ESR spectra as a function of measurement temperature from Fe-doped $\mathrm{Cs}_{2} \mathrm{AgBiBr}_{6}$, in the form of a large ensemble of small single crystals - equivalent to a powder sample, are shown by the red curves in figure 2a. The observed ESR signals are absent in 
undoped $\mathrm{Cs}_{2} \mathrm{AgBiBr}_{6}$ but are found to increase in intensity with increasing $\mathrm{Fe}$ doping concentration in Fe-doped $\mathrm{Cs}_{2} \mathrm{AgBiBr}_{6}$, suggesting their direct association with the $\mathrm{Fe}$ doping. At a temperature $>125 \mathrm{~K}$, the ESR spectra only exhibit a single line with $\mathrm{g} \sim 2.032$. Below $120 \mathrm{~K}$, the single ESR line is replaced by a set of multiple ESR lines with their line spacing increasing with decreasing temperature. The fashion that such multiple ESR lines fan out is characteristic for an ESR spectrum from a high-spin state $(S>1 / 2)$ undergoing increasing zero-field spin splitting as the strength of a low-symmetry crystal field increases ${ }^{25,26}$. To obtain in-depth information on the nature and spin configuration of the responsible paramagnetic centre, the ESR spectra are carefully analysed with the aid of the following effective spin Hamiltonian:

$$
\widehat{H}=g_{e} \mu_{B} \mathbf{B} \widehat{\mathbf{S}}+D(T) S_{z}^{2}+E(T)\left(S_{x}^{2}-S_{y}^{2}\right)
$$

Here, $g_{e}$ is the electron Landé $g$-factor, $\mu_{B}$ the Bohr magneton and $\mathbf{B}$ the magnetic field vector. $\widehat{\boldsymbol{S}}$ is the electron spin operator with components $S_{\mathrm{x}}, S_{\mathrm{y}}$ and $S_{\mathrm{z} .} D(T)$ and $E(T)$ are the temperature dependent axial and rhombic zero-field splitting (ZFS) parameters. From the best fit of the spin Hamiltonian Eq.(1) to the experimental data, the high-spin state observed in ESR at $<120 \mathrm{~K}$ corresponds to the $S=5 / 2$ state. The only plausible candidate that possesses such spin configuration in $\mathrm{Fe}$-doped $\mathrm{Cs}_{2} \mathrm{AgBiBr}_{6}$ is $\mathrm{Fe}^{3+}$ with the $3 \mathrm{~d}^{5}$ configuration. This assignment also identifies $\mathrm{Fe}^{3+}$ to reside on the $\mathrm{M}^{3+}$ site replacing $\mathrm{Bi}^{3+}$, which satisfies the conditions for both chemical bonding and charge neutrality.

The spin Hamiltonian analysis also concludes that $\mathrm{Fe}^{3+}$ experiences a crystal field of axial symmetry, characterized by the spin-Hamiltonian parameters $D>0$ and $E=0$. The nonzero $D$ is responsible for the zero-field spin splitting of the $\mathrm{S}=5 / 2$ states into three Kamer doublets $M_{\mathrm{s}}= \pm \frac{1}{2}, \pm \frac{3}{2}$ and $\pm \frac{5}{2}$, leaving the $\mathrm{M}_{\mathrm{s}}= \pm \frac{1}{2}$ spin sublevel at the lowest energy as illustrated in figure 1d, and the resulting multiple ESR transitions. From a detailed analysis of 
the EPR spectra at various temperatures, the temperature dependent $D(T)$ can be obtained and is plotted in figure $\mathbf{2 b} . D(T)$ is found to increase with decreasing temperature, replicating the temperature dependence of the spontaneous tetragonal strain determined in the early structural analysis of undoped $\mathrm{Cs}_{2} \mathrm{AgBi}(\mathrm{Fe}) \mathrm{Br}_{6}$ by $\mathrm{X}$-ray diffraction ${ }^{24}$. This finding provides a direct proof that the axial crystal field leading to the zero-field splitting of $\mathrm{Fe}^{3+}$ originates from the tetragonal strain of the host crystal itself. It can also be clearly seen from figure $\mathbf{2} \mathbf{b}$ that, when temperature rises towards $120 \mathrm{~K}, D(T)$ approaches zero and remains so at the higher temperatures. This critical temperature coincides with the crystal phase transition of $T_{s} \sim 122 \mathrm{~K}$ from the early studies of X-ray and neutron diffraction ${ }^{24}$. Therefore, we can conclude that the single ESR line observed at $>130 \mathrm{~K}$ in fact stems from the $\mathrm{S}=5 / 2$ spin state of $\mathrm{Fe}^{3+}$ without zero-field splitting $(D=E=0)$ such that all ESR transitions occur at the same resonance field as illustrated in figure 1c. To obtain a more accurate $T_{s}$ from the ESR data, we analyse the temperature dependence of $D(T)$ by the following relation ${ }^{26,27}$;

$$
D(T)\left(\mathrm{Fe}^{3+}\right) \propto\left(T_{s^{-}} T\right)^{\alpha}
$$

The best fit of Eq. 2 to the experimental data yields $T_{s}=122 \mathrm{~K}$, and $\alpha=1 / 2$ for a second-order phase transition based on the mean-field theory ${ }^{27}$. The resulting fitting curve is plotted by the black solid line in figure $\mathbf{2 b}$. The obtained $T_{s}$ value exactly matches that obtained from the Xray and neutron diffraction ${ }^{24}$, demonstrating the power of the ESR spectroscopy in accurately sensing the crystal phase transition in additional to its commonly known capability in retrieving microscopic information on the electronic structure and spin configurations.

We note that, though in principle g-factor anisotropy is also expected in the tetragonal phase, an isotropic $\mathrm{g}$ factor with $\mathrm{g} 2.032$ is deduced within our experimental accuracy throughout the temperature range of $30-300 \mathrm{~K}$ regardless of the crystal phase transition. The observed absence of g-factor anisotropy is rather common for $\mathrm{Fe}^{3+}$ in other materials, as it is 
usually within the experimental error ${ }^{25}$. Using the deduced spin-Hamiltonian parameters, the simulated ESR spectra for powder at different temperatures are also displayed by the grey curves in figure 2a. The excellent agreement between these simulated ESR spectra and the experimental data further justifies our conclusion on the evolution of the $\mathrm{Fe}^{3+}$ spin state accompanying the crystal phase transition of $\mathrm{Cs}_{2} \mathrm{AgBiBr}_{6}$ from the cubic to tetragonal symmetry.

a

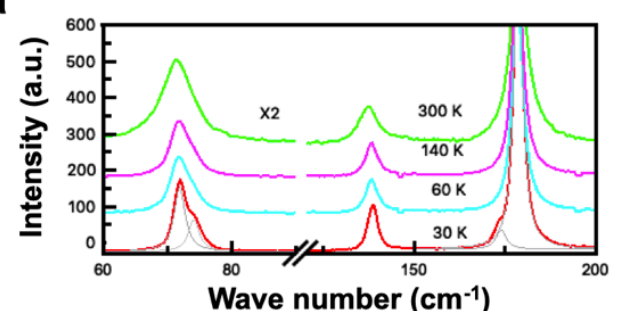

b
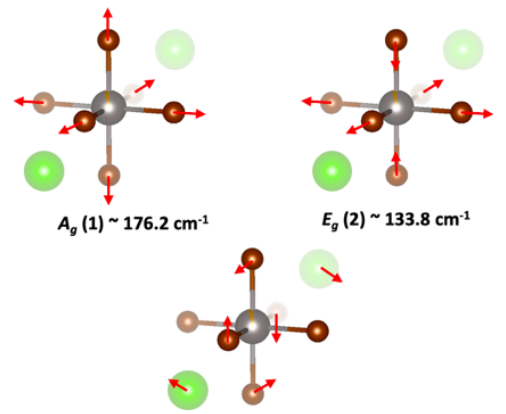

$T_{g}(3) \sim 72 \mathrm{~cm}^{-1}$ c

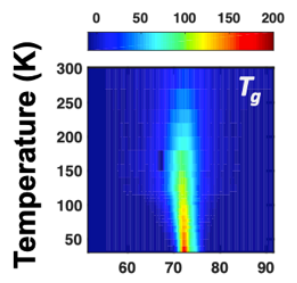

d

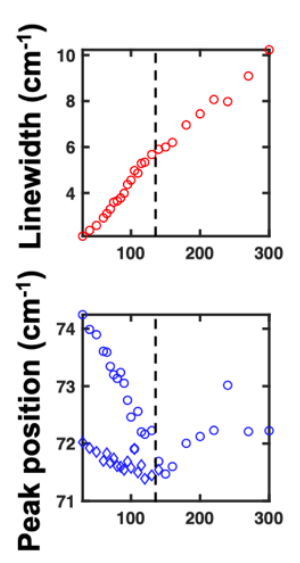

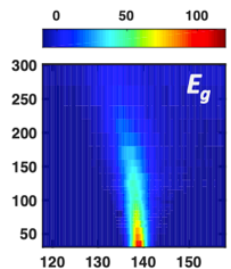

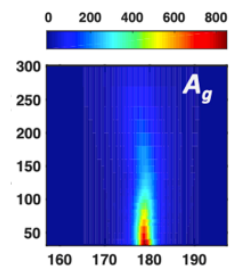

Wave number $\left(\mathrm{cm}^{-1}\right)$
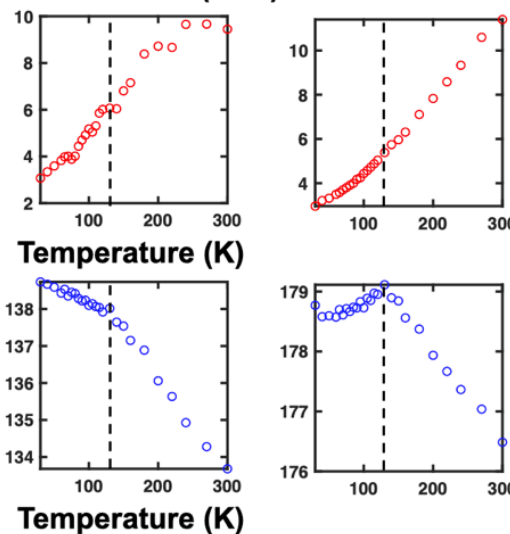

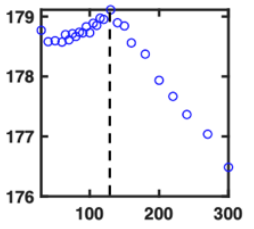

Figure 3 a) Raman spectra at $30 \mathrm{~K}, 60 \mathrm{~K}, 140 \mathrm{~K}$ and $300 \mathrm{~K}$ under the 532-nm laser pumping. Raman Intensity at $300 \mathrm{~K}$ is enlarged by a factor of two for a clear comparison. The Raman signal at $30 \mathrm{~K}$ are fitted with Lorentzian curves in grey. b) Schematic pictures of the dominant Raman modes $A_{g}(1), E_{g}(2)$ and $T_{g}(3)$. c) Contour plot of Raman spectra v.s. temperature of $T_{g}(3), E_{g}(2)$ and $A_{g}(1)$. d) and e) Linewidths and peak positions of these Raman modes as a function of temperature. The dash lines mask the phase transition temperature.

Room-temperature carrier-phonon interactions and the vibrational modes associated with the $\mathrm{MBr}_{6}$ octahedra in $\mathrm{Cs}_{2} \mathrm{AgBiBr}_{6}$ were reported early and a strong Fröhlich interaction was suggested $\mathrm{d}^{22,23,28}$. The dominant Raman modes of the cubic phase have been identified at 75,135 and $175 \mathrm{~cm}^{-1}$ that belong to $T_{g}$ (enharmonic breathing), $E_{g}$ and $A_{g}$ (both stretching) modes $^{23,28}$ of either $\left(\mathrm{BiBr}_{6}\right)^{3-}$ octahedra ${ }^{28}$ or $\left(\mathrm{AgBr}_{6}\right)^{5-}$ octahedra $^{23}$, as schematically illustrated in figure 3b. The subscript $g$ refers to a Raman active mode. Up to now, nothing is known about the vibrational modes of the tetragonal $\mathrm{Cs}_{2} \mathrm{AgBiBr}_{6}$ after the crystal phase transition. 
In principle, a crystal-symmetry reduction from $\mathrm{Fm} \overline{3} \mathrm{~m}$ to $\mathrm{I} / \mathrm{m}$ is also expected to lift the degeneracy of some vibrational modes associated with the octahedral sublattices. For example, the three-fold degenerate Raman active mode of $T_{g}(3)$ is predicted to split into $E_{g}(2)+A_{g}(1)$ based on group theory (see figure 1e and 1f). Here, the number in each parenthesis refers to the degeneracy of the modes.

Apart from the three Raman modes of the $\mathrm{MBr}_{6}$ octahedra, the crystal symmetries also allow Raman scattering of low-frequency lattice modes, as was observed in elpasolites ${ }^{29-31}$. The absence of such Raman modes in our study could possibly be due to their low frequencies (< $50 \mathrm{~cm}^{-1}$ ) that are beyond the range of our instrumental coverage.

To evaluate the influence of the crystal phase transition, we studied the dominant Raman modes over a wide temperature range of 30-300 K. Figure 3a shows representative Raman spectra measured at $30 \mathrm{~K}, 60 \mathrm{~K}, 140 \mathrm{~K}$ and $300 \mathrm{~K}$, taken as an example from the Fe-doped $\mathrm{Cs}_{2} \mathrm{AgBiBr}_{6}$ crystal along a $<111>$ crystallographic axis. At room temperature three Raman modes are observed that agree with the previously identified modes of $T_{g}(3) \sim 72 \mathrm{~cm}^{-1}, E_{g}(2)$ $\sim 133.8 \mathrm{~cm}^{-1}$ and $A_{g}(1) \sim 176.2 \mathrm{~cm}^{-1}$ from the undoped $\mathrm{Cs}_{2} \mathrm{AgBiBr}_{6}$, showing that they originate from the vibrational modes of the pristine crystal that are unaffected by the Fe doping.

An overview of the temperature dependence of these three Raman modes is given in figure 3c, whereas their linewidths and peak positions deduced from the fitting with Lorentzian lines are shown in figure 3d and 3e. For all modes, the linewidths monotonically decrease from $\sim 10 \mathrm{~cm}^{-1}$ towards $\sim 3 \mathrm{~cm}^{-3}$, with an apparent change of the slope near $T_{\mathrm{s}}$ for the $T_{g}(3)$ mode. We tentatively attribute this linewidth narrowing of the Raman modes to a reduced contribution of thermal bath. In contrast, the peak positions of all three Raman modes exhibit striking variations near $T_{\mathrm{s}}$. For the $T_{g}(3)$ mode, it splits into two components at $T<T_{\text {s }}$, i.e. $E_{g}(2)+$ 
$A_{g}(1)$ as predicted by group theory. The magnitude of the splitting is proportional to the strength of the tetragonal strain. Furthermore, the peak position of $T_{g}(3)$ in the cubic phase displays an anomalous behaviour of mode softening, namely, its frequency reduces from $\sim 72.5$ $\mathrm{cm}^{-1}$ to $\sim 71.4 \mathrm{~cm}^{-1}$ upon cooling down towards $T_{s}$. This frequency reduction is in fact opposite to what is usually expected from hardening (i.e. a frequency increase) of a Raman mode accompanying by shrinking of lattice constants upon cooling, as seen for $E_{g}(2) \sim 133.8 \mathrm{~cm}^{-1}$ and $A_{g}(1) \sim 176.2 \mathrm{~cm}^{-1}$ to be discussed below. The observed frequency reduction of $T_{g}(3)$ when temperature approaches $T_{s}$ from both cubic and tetragonal phases, on the other hand, resembles a 'soft-phonon' lattice mode ${ }^{32}$. This finding might indicate a link between the $T_{g}(3)$ mode and the lattice vibrations that are important to the phase transition.

In contrast to $T_{g}(3)$, both $E_{g}(2) \sim 133.8 \mathrm{~cm}^{-1}$ and $A_{g}(1) \sim 176.2 \mathrm{~cm}^{-1}$ exhibit the character of hard Raman modes. In the cubic phase, their frequencies increase with decreasing temperature from 133.8 to $138.1 \mathrm{~cm}^{-1}$ and from 176.2 to $179.1 \mathrm{~cm}^{-1}$, respectively (see figure 3e), as expected from symmetric shrinking of lattice parameters that increases total energy of the symmetric octahedral vibrational modes. This trend discontinues at $T_{\mathrm{s}}$. For $A_{g}(1) \sim 176.2$ $\mathrm{cm}^{-1}$ mode, the mode frequency becomes lower as temperature further decreases. This could be understood by considering an asymmetric modification of the $\mathrm{MBr}_{6}(\mathrm{M}=\mathrm{Ag}$ or $\mathrm{Bi})$ bond length below $T_{s}$. For the $\mathrm{AgBr}_{6}$ octahedra, the bond length in the ab plane $\left(\mathrm{Ag}-\mathrm{Br}_{\mathrm{a}, \mathrm{b}}\right)$ gradually changes from 2.8 to $2.785 \AA(\sim 0.53 \%)$ as temperature reduces from 130 to $30 \mathrm{~K}$, whilst the bond length of $\mathrm{Ag}-\mathrm{Br}_{\mathrm{c}}$ increases from 2.8 to $2.838 \AA(1.35 \%)$. This translates to an increase of the effective octahedral volume and, therefore, a decrease of the $A_{g}(1)$ mode frequency below $T_{\mathrm{s}}$. For the $E_{g}(2) \sim 133.8 \mathrm{~cm}^{-1}$ mode, on the other hand, it keeps hardening below $T_{\mathrm{s}}$ but at a lower rate. This difference in the temperature dependence between $A_{g}(1)$ and $E_{g}(2)$ below $T_{s}$ may stem from the fact that the $E_{g}(2)$ mode transforms into $A_{g}(1)+B_{g}(1)$ modes and the frequency 
difference between these two modes cannot be simply predicted under the effective volume consideration. Even at $30 \mathrm{~K}$, the splitting of $E_{g}(2)$ remains unresolved, possibly due to a large Raman linewidth.

There is an appearance of a weak satellite Raman mode at $172 \mathrm{~cm}^{-1}$ near the shoulder of the main $178.7 \mathrm{~cm}^{-1} A_{g}(1)$ at $30 \mathrm{~K}$ in both Fe-doped and undoped samples. This mode resembles that seen in the low-temperature Raman spectra from $\mathrm{Rb}_{2} \mathrm{KScF}_{6}$ crystals in the monoclinic phase $\mathrm{s}^{30}$, where such a transition belongs to a formally inactive $\mathrm{X}(0,0, \pi / a)$ Raman mode in the cubic phase that becomes allowed in the monoclinic phase with a double primitive cell volume. From the previous XRD analysis of undoped $\mathrm{Cs}_{2} \mathrm{AgBiBr}_{6}{ }^{24}$ and also our own XRD analysis of $\mathrm{Cs}_{2} \mathrm{AgBi}(\mathrm{Fe}) \mathrm{Br}_{6}$ at $30 \mathrm{~K}$, the crystal symmetry deduced from the Rietveld refinement remains to be the space group $\mathrm{I} 4 / \mathrm{m}$ of tetragonal symmetry. At present, the exact origin of the Raman mode at $172 \mathrm{~cm}^{-1}$ remains unknown, which calls for further careful structural studies of $\mathrm{Cs}_{2} \mathrm{AgBiBr}_{6}$ at and below $30 \mathrm{~K}$.

In summary, we have shown from the ESR and Raman spectroscopies that the crystal phase transition of the lead-free double perovskite $\mathrm{Cs}_{2} \mathrm{AgBiBr}_{6}$ has a profound effect on the high-spin states of e.g. a transitional metal ion $\mathrm{Fe}^{3+}$ (residing on the $\mathrm{Bi}$ site) and the vibrational modes. The structural phase transition predominantly manifests itself as a source of symmetrybreaking that lifts the degeneracy of the spin states and Raman modes when the crystal undergoes a transition from the cubic to tetragonal phase. This splits the 6-fold degenerate $\mathrm{S}=5 / 2$ state of $\mathrm{Fe}^{3+}$ to three Kramer doublets $\mathrm{M}_{\mathrm{s}}= \pm \frac{1}{2}, \pm \frac{3}{2}$ and $\pm \frac{5}{2}$ at zero magnetic field, thereby transforming the corresponding ESR spectra from a single line to a set of multiple lines. The size of the zero-fielding spin splitting is shown to directly correlate with the strength of the tetragonal strain field. A similar symmetry-reduction effect is found for the enharmonic breathing mode $T_{g}(3)$ of the $\mathrm{MBr}_{6}$ octahedra, which splits into $E_{g}(2)+A_{g}(1)$ below the phase 
transition temperature $T_{s}$ with the extent of the splitting scaling with the tetragonal strain field. Anomalous softening of the $T_{g}(3)$ mode near $T_{s}$ is observed, unlike the stretching modes $E_{g}$ and $A_{g}$ of the $\mathrm{MBr}_{6}$ octahedra that exhibit expected hardening when the temperature decreases. The observed high sensitivity of the ESR and Raman spectroscopies to a small degree of lattice distortion in turn demonstrates the power of these techniques in probing structural phase transitions and in providing in-depth information on the interplay between the structural and spin properties of lead-free double perovskites - a newly-emerging and promising class of materials relevant to low-cost and high-efficiency photovoltaic and optoelectronic applications. 


\section{Acknowledgement}

Y.P. acknowledges a starting grant from the Swedish Research Council (VR-2017-05285). I.A.B and W.M.C acknowledge the financial support by Linköping University through the Professor Contracts and Swedish Government Strategic Research Area in Materials Science on Functional Materials at Linköping University (Faculty Grant SFO-Mat-LiU No 2009 00971). F.G. and W.M.C. acknowledge the support from the Knut and Alice Wallenberg Foundation (Dnr KAW 2019.0082).

\section{Methods}

Sample preparation - Solid $\mathrm{CsBr}(213 \mathrm{mg}, 1.00 \mathrm{mmol}), \mathrm{BiBr}^{3}(135 \mathrm{mg}, 0.3 \mathrm{mmol})$ and $\mathrm{FeBr}^{3}$ (59.1 mg, $0.2 \mathrm{mmol}$ ) were dissolved in $4 \mathrm{~mL}$ of 47\% HBr. Solid $\mathrm{AgBr}$ (94 mg, $0.5 \mathrm{mmol}$ ) was then added. The mixed solution was transferred to Teflon-lined digestion bomb and placed in the oven where it was heated at $120{ }^{\circ} \mathrm{C}$ for $24 \mathrm{~h}$. The crystal of Fe-doped $\mathrm{Cs}_{2} \mathrm{AgBiBr}_{6}$ was then formed after the mixture was slowly cooled down to room temperature.

ESR - ESR was performed with a Bruker Elexsys E500 spectrometer operating at about 9.3 GHz. ESR spectra were recorded in dark. Crystal powder was prepared via gliding several Fedoped or undoped $\mathrm{Cs}_{2} \mathrm{AgBiBr}_{6}$ single crystals into a large number of small pieces. The powder was sealed in an evacuated quartz tube and placed in a He-flow cryostat.

Raman spectroscopy - Raman measurements were carried out using a confocal Horiba JobinYvon HR800 system. A 1800 1/mm single-grating monochromator in the conjunction with a Si charge-coupling-device (CDD) array (1024 by 256-pixel counts) were used. This translated to a spectral resolution better than $1 \mathrm{~cm}^{-1}$, with pixel counting of 4 pixels $/ \mathrm{cm}^{-1}$. A solid-state diode laser with a wavelength of $532 \mathrm{~nm}$ was used as a pumping source, with power below $1 \mathrm{~mW} \mu \mathrm{m}^{-}$ ${ }^{2}$ to avoid sample heating. $532 \mathrm{~nm}$ edge filters (Semrock LE03-532RE-25 with edge steepness of $37.5 \mathrm{~cm}^{-1}$ ) were used to filter out the laser line. The edge filter and the single-grating 
monochromator limited the detection of Raman modes with wave numbers below $50 \mathrm{~cm}^{-1}$. The induced Raman scattering signals were collected via a $50 \times$ objective with $\mathrm{NA}=0.5$ in a backscattering geometry. The light beam was directed parallel to a $<111>$ crystallographic direction of an undoped or $\mathrm{Fe}$-doped $\mathrm{Cs}_{2} \mathrm{AgBiBr}_{6}$ single crystal. The samples were mounted in a He flow cryostat. Temperature stability was better than $0.5 \mathrm{~K}$. 


\section{References}

1. Jena, A. K., Kulkarni, A. \& Miyasaka, T. Halide Perovskite Photovoltaics: Background, Status, and Future Prospects. Chem. Rev. 119, 3036-3103 (2019).

2. Nayak, P. K., Mahesh, S., Snaith, H. J. \& Cahen, D. Photovoltaic solar cell technologies: analysing the state of the art. Nat. Rev. Mater. 4, 269-285 (2019).

3. NREL best Research-Cell Efficiency Chart. (2020).

4. Stranks, S. D. \& Snaith, H. J. Metal-halide perovskites for photovoltaic and lightemitting devices. Nat. Nanotechnol. 10, 391 (2015).

5. Zhang, W., Eperon, G. E. \& Snaith, H. J. Metal halide perovskites for energy applications. Nat. Energy 1, 16048 (2016).

6. Sutherland, B. R. \& Sargent, E. H. Perovskite photonic sources. Nat. Photonics 10, 295 (2016).

7. Sheng, R. et al. Monolithic Wide Band Gap Perovskite/Perovskite Tandem Solar Cells with Organic Recombination Layers. J. Phys. Chem. C 121, 27256-27262 (2017).

8. Nogay, G. et al. 25.1\%-Efficient Monolithic Perovskite/Silicon Tandem Solar Cell Based on a p-type Monocrystalline Textured Silicon Wafer and High-Temperature Passivating Contacts. ACS Energy Lett. 4, 844-845 (2019).

9. Manser, J. S., Saidaminov, M. I., Christians, J. A., Bakr, O. M. \& Kamat, P. V. Making and Breaking of Lead Halide Perovskites. Acc. Chem. Res. 49, 330-338 (2016).

10. Babayigit, A., Ethirajan, A., Muller, M. \& Conings, B. Toxicity of organometal halide perovskite solar cells. Nat. Mater. 15, 247 (2016).

11. Giustino, F. \& Snaith, H. J. Toward Lead-Free Perovskite Solar Cells. ACS Energy Lett. 1, 1233-1240 (2016).

12. Slavney, A. H., Hu, T., Lindenberg, A. M. \& Karunadasa, H. I. A Bismuth-Halide Double Perovskite with Long Carrier Recombination Lifetime for Photovoltaic Applications. J. Am. Chem. Soc. 138, 2138-2141 (2016).

13. Du, K., Meng, W., Wang, X., Yan, Y. \& Mitzi, D. B. Bandgap Engineering of LeadFree Double Perovskite Cs2AgBiBr6 through Trivalent Metal Alloying. Angew. Chemie Int. Ed. 56, 8158-8162 (2017).

14. Volonakis, G. et al. Cs2InAgC16: A New Lead-Free Halide Double Perovskite with Direct Band Gap. J. Phys. Chem. Lett. 8, 772-778 (2017).

15. Greul, E., Petrus, M. L., Binek, A., Docampo, P. \& Bein, T. Highly stable, phase pure Cs2AgBiBr6 double perovskite thin films for optoelectronic applications. J. Mater. Chem. A 5, 19972-19981 (2017).

16. Igbari, F. et al. Composition Stoichiometry of Cs2AgBiBr6 Films for Highly Efficient Lead-Free Perovskite Solar Cells. Nano Lett. 19, 2066-2073 (2019).

17. Ning, W. \& Gao, F. Structural and Functional Diversity in Lead-Free Halide Perovskite Materials. Adv. Mater. 31, 1900326 (2019).

18. Pan, W. et al. Cs2AgBiBr6 single-crystal X-ray detectors with a low detection limit. Nat. Photonics 11, 726-732 (2017). 
19. Luo, J. et al. Efficient and stable emission of warm-white light from lead-free halide double perovskites. Nature 563, 541-545 (2018).

20. Ning, W. et al. Thermochromic Lead-Free Halide Double Perovskites. Adv. Funct. Mater. 29, 1807375 (2019).

21. Ning, W. et al. Long Electron-Hole Diffusion Length in High-Quality Lead-Free Double Perovskite Films. Adv. Mater. 30, 1706246 (2018).

22. Kentsch, R. et al. Exciton Dynamics and Electron-Phonon Coupling Affect the Photovoltaic Performance of the Cs2AgBiBr6 Double Perovskite. J. Phys. Chem. C 122, 25940-25947 (2018).

23. Steele, J. A. et al. Giant Electron-Phonon Coupling and Deep Conduction Band Resonance in Metal Halide Double Perovskite. ACS Nano 12, 8081-8090 (2018).

24. Schade, L. et al. Structural and Optical Properties of Cs2AgBiBr6 Double Perovskite. ACS Energy Lett. 4, 299-305 (2019).

25. Abragam, A. \& Bleaney, B. Electron paramagnetic resonance of transition ions, . (Oxford University Press, Oxford, 1970).

26. Owens, F. J., Poole, C. P. \& Farach, H. A. Magnetic Resonance of Phase Transitions. (Academic Press, 2013).

27. Thomas, H. \& Müller, K. A. Structural Phase Transitions in Perovskite-Type Crystals. Phys. Rev. Lett. 21, 1256-1259 (1968).

28. Zelewski, S. J. et al. Revealing the nature of photoluminescence emission in the metalhalide double perovskite Cs2AgBiBr6. J. Mater. Chem. C 7, 8350-8356 (2019).

29. Vtyurin, A., Krylov, A., Voronov, V. \& Krylova, S. Raman scattering and phase transitions in fluorides with elpasolite structure. Ferroelectrics 512, 58-64 (2017).

30. Krylov, A. S., Vtyurin, A. N., Bulou, A. \& Voronov, V. N. Raman Spectra and Phase Transitions in the Rb 2 KScF 6 Elpasolite. Ferroelectrics 284, 47-64 (2003).

31. Krylov, A. S., Vtyurin, A. N., Oreshonkov, A. S., Voronov, V. N. \& Krylova, S. N. Structural transformations in a single-crystal Rb2NaYF6: Raman scattering study. $J$. Raman Spectrosc. 44, 763-769 (2013).

32. Scott, J. F. Soft-mode spectroscopy: Experimental studies of structural phase transitions. Rev. Mod. Phys. 46, 83-128 (1974). 


\section{TOC/abstract graphics}
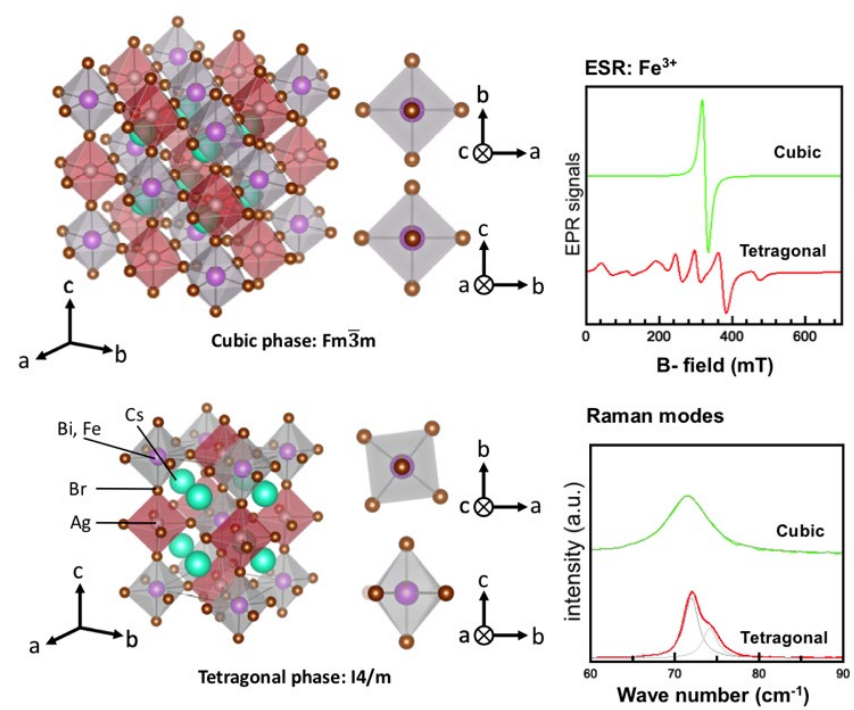